\title{
Robotic resection of a giant retroperitoneal leiomyosarcoma: A case report
}

\author{
MARINOS C. MAKRIS ${ }^{1,2}$, PANOS G. ATHANASOPOULOS ${ }^{3}$, MICHAEL KORNAROPOULOS ${ }^{1}$, \\ PERICLIS CHRYSOCHERIS ${ }^{3}$, FOTIOS ANTONAKOPOULOS ${ }^{3}$, ANTONIA MATHIOULAKI ${ }^{3}$, \\ DIAMANTIS I. TSILIMIGRAS ${ }^{4}$, ARGYRIOS IOANNIDIS ${ }^{5}$, MICHAEL K. KONSTANTINIDIS ${ }^{4}$, \\ DIMITRIOS MORIS $^{4}$ and KONSTANTINOS M. KONSTANTINIDIS ${ }^{3}$
}

${ }^{1}$ First Surgical Department, General Hospital of Athens 'G. Gennimatas', Athens 11527;

${ }^{2}$ Alpha Institute of Biomedical Sciences (AIBS), Athens 15123; ${ }^{3}$ Department of General, Laparoscopic,

Robotic and Bariatric Surgery, Athens Medical Center, Athens $15125 ;{ }^{4}$ School of Medicine, National and Kapodistrian University of Athens, Athens 11527; ${ }^{5}$ Second Surgical Department, Sismanoglion General Hospital, Athens 15126, Greece

Received October 20, 2018; Accepted April 11, 2019

DOI: $10.3892 / \mathrm{mco} .2019 .1928$

\begin{abstract}
The management of abdominal leiomyosarcoma is challenging. Surgical excision is considered the only effective treatment; however, this is associated with considerable morbidity. Robotic surgery has emerged during the past decades and has enhanced the general surgery armamentarium, allowing surgeons to carry out demanding operations in a safe manner. The surgical resection of retroperitoneal leiomyosarcoma (RPLM) can be associated with significant morbidity, which is primarily due to the origin or the close proximity of the tumor with important vascular structures, including the inferior vena cava and tributaries, the duodenum and the ureter. The present case describes the first case of robotic resection of RPLM in a high-volume robotic center. In the present case, a large RPLM was safely removed with respect to oncological principles with the use of the Da Vinci platform.
\end{abstract}

\section{Introduction}

The utilization of robotic systems in surgery has emerged as an alternative to open and laparoscopic surgery with the preliminary results being promising to date (1). Initially used in urologic operations (2) and cholecystectomies (3), the application of the Da Vinci platform has lately expanded to more challenging cases such as aneurysm repair (4) and liver (5) and pancreatic resections (6). The advantages of this technology include better visibility via three-dimensional (3D)

Correspondence to: Dr Marinos C. Makris, First Surgical Department, General Hospital of Athens 'G. Gennimatas', 154 Mesogeion Avenue, Athens 11527, Greece

E-mail:m.makris@aibs.gr

Key words: robotic resection, Da Vinci, retroperitoneal leiomyosarcoma imaging, stable field, enhanced maneuverability due to articulated instruments and greater precision in the dissection of tissues (7).

Retroperitoneal sarcomas (RPS) are malignant neoplasms that account for $10-15 \%$ of all soft-tissue tumors (8). Histological tumor type, grade, location and extension of resection have been well-documented prognostic factors for long-term survival. Given the complex nature of RPS surgery, much emphasis has recently been placed on the appropriate referral and management of these patients at specialized centers in order to ensure high quality of surgical care (9).

Herein, we present the first described case of robotic resection of a retroperitoneal leiomyosarcoma (RPLM) in a high-volume center for robotic operations.

\section{Case report}

A 72-year-old male was admitted due to progressive abdominal distension, dull abdominal pain for the last 3 months, constitutional symptoms and constipation. Physical examination revealed a slightly distended abdomen and an abdominal mass in right side which appeared hard and immobile.

The patient subsequently underwent an abdominal and pelvic computed tomography (CT) (Fig. 1A) and magnetic resonance imaging (MRI) (Fig. 1B) which revealed a solid heterogeneous lesion $12,1 \times 11,8 \times 15 \mathrm{~cm}$ located from the descending part of the duodenum and head of the pancreas to transverse mesocolon and a second smaller lesion $3 \times 2,7 \mathrm{~cm}$ infiltrating the ascending colon. No major vessels were invaded and no liver metastases were reported.

The patient was scheduled for elective robotic tumor excision after acknowledging the risks of this challenging operation and the benefits derived from utilizing the robotic system in this particular occasion.

Intraoperatively, a gigantic retroperitoneal mass was revealed compressing the duodenum and the pancreatic groove, yet did not invade it. The second smaller mass was infiltrating the ascending colon. The mass was dissected from 

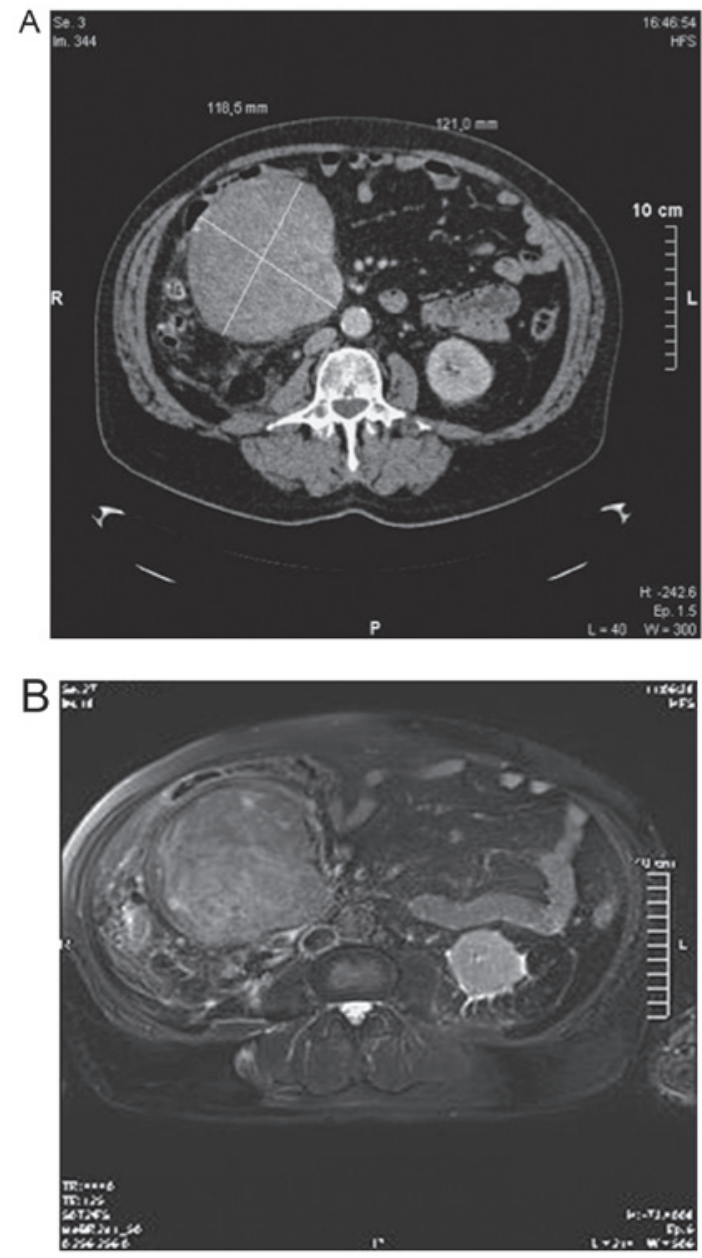

Figure 1. (A) Computerized tomography of the abdomen and pelvis with intravenous contrast demonstrating the retroperitoneal mass and its proximity with the aorta and the inferior vena cava. (B) Magnetic resonance imaging of the abdomen and pelvis and T2 sequence revealed the mass. No invasion of the aorta and inferior vena cava is noted

the duodenum, head of the pancreas and the right kidney using sharp and blunt dissection without any breach of tumor capsule. After dissection, the tumor was extracted completely. An upper midline incision was performed and a right hemicolectomy was performed (Fig. 2). A frozen section was sent from the margin of remaining tissue, which was negative for malignancy. Meticulous hemostasis was performed to ensure no residual bleeding. The operative time was $7.5 \mathrm{~h}$ including the robotic docking time of $30 \mathrm{~min}$. There were no intraoperative or postoperative complications and the patient was discharged home on the 4th postoperative day.

The histopathology report described a 19x17,5 cm retroperitoneal pleomorphic leiomyosarcoma, grade 3 , mitotic index of 10 mitoses per 50 high power fields, CD 117 [C-KIT (-)], CD $68, \mathrm{Ki} 67(\mathrm{MIBI})(+)$. The surgical margins were free of disease. Postoperatively the patient was evaluated by medical oncology and radiation oncology.

\section{Discussion}

Patients with RPS are usually diagnosed late, when the tumor is already of considerable size and close or attached to critical retroperitoneal structures and organs. This physical history

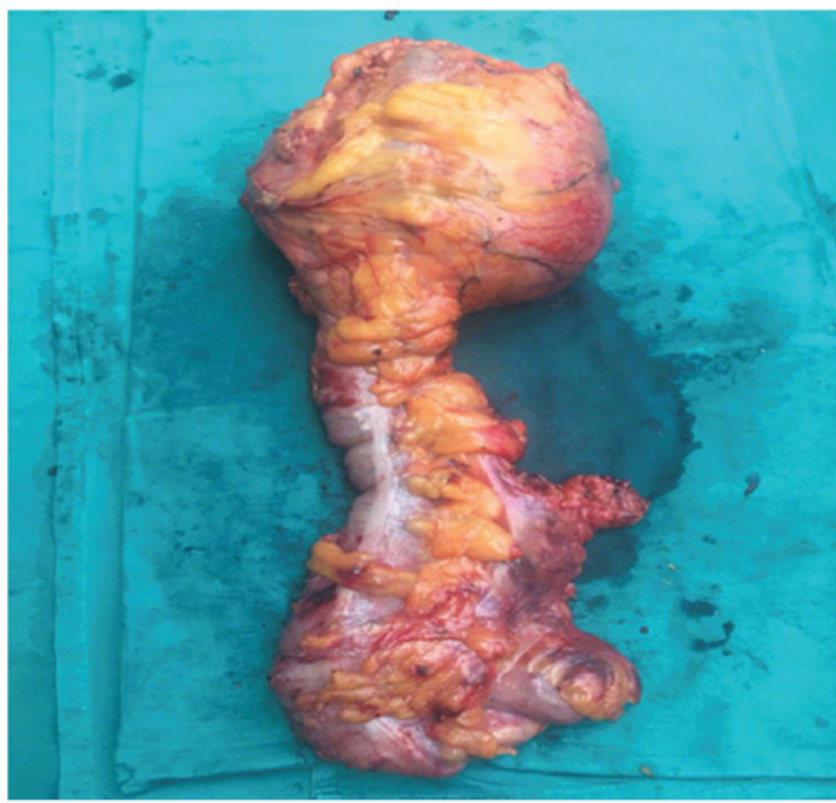

Figure 2. Surgical specimen following robotic excision.

limits the ability of surgeons to perform wide resections with clear margins. Importantly, incomplete surgical resection comprises one of the greatest risk factors locoregional recurrence and poor survival (10).

The management of bulky intra-abdominal extra-luminal tumors is challenging due to their close proximity or diffusion of other structures. The surgical resection of RPLM can be associated with significant morbidity given that they usually invade important vascular structures such as the inferior vena cava (IVC) and tributaries, the duodenum and the ureter (11). The intraoperative 30-day mortality rate has been reported to be as high as $15 \%$ in resections of RPLMs arising from the IVC (12). However, a curative intent operation entails a wide resection incorporating the tumor and all the involved structures (e.g duodenum, colon, kidney, IVC) in order to achieve adequate resection margins (13).

Robotic surgery is a minimally invasive approach that has gained significant popularity over the last decade. Advantages of this procedure include less intraoperative blood loss, better visualization, shorter hospital stay, and rapid patient recovery (14). Importantly, thanks to the highly articulated instruments and the increased precision in surgical manipulation, the robotic system is even more important in cases requiring fine dissection, such as cases invading the IVC or the aorta (5). Although in our case the patient recovered rapidly, uneventfully, after a resection with clear margins, there is a paucity of data on the oncologic outcomes of such operations in large series; yet preliminary results in several cancer centers are promising (15).

In this report, we describe the utilization of the robotic $\mathrm{Da}$ Vinci platform by our experienced team made the resection of the giant tumor feasible without limiting the oncologic outcome of the operation. The three-dimensional imaging system was beneficial in understanding the relationship of the mass to the ureter, aorta and vena cava as well as colon and small bowel. Further reports are needed to elucidate the cases that can take advantage of the robotic technology. 


\section{Acknowledgements}

Not applicable.

\section{Funding}

No funding was received.

\section{Availability of data and materials}

The datasets used and/or analyzed during the present study are available from the corresponding author on reasonable request.

\section{Authors' contributions}

MCM, PGA, MK, PC, FA, AM, DIT, KMK and AI conceived and designed the present work. MKK, KMK and DM performed the data analysis. MCM and KMK drafted the manuscript. MCM, PGA, MK, PC, FA, AM, DIT, AI, MKK, $\mathrm{DM}$ and $\mathrm{KMK}$ critically revised the manuscript, provided approval of the final version and agreed to be accountable for all aspects of the work.

\section{Ethics approval and consent to participate}

The study was conducted according to the Declaration of Helsinki.

\section{Patient consent for publication}

Written informed consent was obtained from the patient for publication of this Case Report/any accompanying images.

\section{Competing interests}

The authors declare that they have no competing interests.

\section{References}

1. Hazey JW and Melvin WS: Robot-assisted general surgery. Semin Laparosc Surg 11: 107-112, 2004.

2. Vince R, Hampton LJ, Vartolomei MD, Shariat SF, Porpiglia F and Autorino R: Robotic assisted simple prostatectomy: Recent advances. Curr Opin Urol 28: 309-314, 2018.
3. Angelou A, Skarmoutsos A, Margonis GA, Moris D, Tsigris C and Pikoulis E: Robotic single port cholecystectomy: Current data and future perspectives. Minerva Chir 72: 140-145, 2017.

4. Makris MC, Moris D, Papalouca K, Malietzis G and Makris GC The current status of robotic vascular surgery in the abdominal cavity. Int Angiol 35: 1-7, 2016.

5. Tsilimigras DI, Moris D, Vagios S, Merath K and Pawlik TM: Safety and oncologic outcomes of robotic liver resections: A systematic review. J Surg Oncol 117: 1517-1530, 2018.

6. Kornaropoulos M, Moris D, Beal EW, Makris MC, Mitrousias A, Petrou A, Felekouras E, Michalinos A, Vailas M, Schizas D and Papalampros A: Total robotic pancreaticoduodenectomy: A systematic review of the literature. Surg Endosc 31: 4382-4392, 2017.

7. Konstantinidis KM, Hiridis S and Karakitsos D: Robotic-assisted surgical removal of pelvic schwannoma: A novel approach to a rare variant. Int J Med Robot 7: 55-59, 2011.

8. von Mehren M, Randall RL, Benjamin RS, Boles S, Bui MM, Conrad EU III, Ganjoo KN, George S, Gonzalez RJ, Heslin MJ, et al: Soft tissue sarcoma, version 2.2016, NCCN clinical practice guidelines in oncology. J Natl Compr Canc Netw 14: 758-786, 2016.

9. Moris D, Petrou A, Papalampros A, Tsilimigras DI and Felekouras E: Retroperitoneal sarcomas: Does the center really matter? Surgery 163: 971-972, 2018

10. Stahl JM, Corso CD, Park HS, An Y, Rutter CE, Han D and Roberts KB: The effect of microscopic margin status on survival in adult retroperitoneal soft tissue sarcomas. Eur J Surg Oncol 43: 168-174, 2017.

11. Petrou A, Constantinidou A, Kontos M, Papalampros A, Moris D, Bakoyiannis C, Neofytou K, Kourounis G and Felekouras E: Comprehensive surgical treatment as the mainstay of management in retroperitoneal sarcomas: Retrospective study from two non-sarcoma specialist centers. Anticancer Res 37: 2025-2031, 2017.

12. Konofaos P, Spartalis E, Moris D, Athanasiou A, Dimitroulis D, Markakis C, Kostakis ID, Nikiteas N and Kouraklis G: Challenges in the surgical treatment of retroperitoneal sarcomas. Indian J Surg 78: 1-5, 2016.

13. Trans-Atlantic RPS Working Group: Management of recurrent retroperitoneal sarcoma (RPS) in the adult: A consensus approach from the trans-atlantic RPS working group. Ann Surg Oncol 23: 3531-3540, 2016.

14. Jayne D, Pigazzi A, Marshall H, Croft J, Corrigan N, Copeland J, Quirke P, West N, Rautio T, Thomassen N, et al: Effect of robotic-assisted vs. conventional laparoscopic surgery on risk of conversion to open laparotomy among patients undergoing resection for rectal cancer: The rolarr randomized clinical trial. JAMA 318: 1569-1580, 2017.

15. Holmer C and Kreis ME: Systematic review of robotic low anterior resection for rectal cancer. Surg Endosc 32: 569-581, 2018. 